\title{
New technique for reconstructing instantaneous velocity profiles from viscometric tests: Application to pasty materials
}

\author{
J. C. Baudez \\ Cemagref, Domaine des Palaquins, 03150 Montoldre, France \\ S. Rodts, X. Chateau, and P. Coussot ${ }^{\mathrm{a})}$ \\ Laboratoire des Matériaux et des Structures du Génie Civil, \\ Champs sur Marne, France
}

(Received 13 June 2003; final revision received 8 September 2003)

\section{Synopsis}

We present a new technique for reconstructing the instantaneous velocity profiles during creep, dynamic, or ramp tests under controlled stress in wide-gap Couette flows, from a series of similar tests under smaller stress amplitudes. This approach is based on a rigourous theory, and since it requires that the fluid does not flow close to the outer cylinder, it is particularly suitable for yield stress fluids. The interest of this reconstruction technique is that it is simpler than direct techniques (nuclear magnetic resonance, light scattering, particle imaging velocimetry, etc.) and has almost no limitations in time and space resolution. Thus, one can obtain the velocity profiles under steady-state and transient flows. We show that for a commercial hair gel the velocity profile obtained with this technique is in excellent agreement with that found from magnetic resonance imaging rheometry within the range of measurement (four decades of velocity). From other tests with a mustard and a kaolin-water suspension we demonstrate that the "viscosity bifurcation" effect observed with various pasty materials [Coussot et al., Phys. Rev. Lett. (2002a)] is directly associated with an abrupt change in the slope of the velocity profiles at the interface between the sheared and unsheared regions. We also show that the effect of wall slip on the reconstructed velocity profile is to shift the level of the unsheared region to a virtual, constant, finite, velocity level. (C) 2004 The Society of Rheology. [DOI: 10.1122/1.1626677]

\section{INTRODUCTION}

Recently, it has been shown that many pasty materials (muds, clays, cement, paints, concentrated emulsions, foams, granular materials) cannot flow steadily at a rate smaller than a critical value [Coussot et al. (2002a, 2002b); Da Cruz et al. (2002); Walls et al. (2003)]:

(i) When the applied stress is smaller than a critical value $\left(\tau_{c}\right)$, the viscosity continuously increases in time and, in some cases, the material may eventually stop flowing.

(ii) When the applied stress is slightly larger than $\tau_{c}$, the viscosity continuously

\footnotetext{
a) Author to whom correspondence should be addressed; electronic mail: philippe.coussot@lcpc.fr
} 
decreases in time and the flow rate accelerates until reaching a steady value always larger than a finite value.

This result was interpreted as a flow instability occurring when the effective shear rate of the material falls below a critical value. However, this relies on macroscopic measurements and thus remains subject to other interpretations assuming specific local characteristics of the flow within the gap (slip, fracture, density heterogeneities, etc.). Direct observations of the flow field in a transparent material or by magnetic resonance imaging (MRI) rheometry in various pasty materials remaining homogeneous could nevertheless confirm our first interpretation and provide more quantitative results: in a Couette geometry there is a discontinuity in the slope of the velocity profile at the interface between the sheared and the unsheared regions [Raynaud et al. (2002); Coussot et al. (2002)]; in a cone-and-plate geometry, at sufficient low apparent shear rates, the sheared thickness decreases with the relative rotation velocity while the shear rate in the sheared region remains almost constant [Pignon et al. (1996); Coussot et al. (2002)]; in both cases there is effectively no steady flow at shear rates ranging from zero to a finite, critical value.

There is a somewhat similar issue for various solutions of surfactants forming onion or wormlike micelles [see, for example, Vermant (2001) for a more complete review]: in their flow curve a stress plateau, separating two regions of significant increase in stress, has been observed [Rehage and Hoffmann (1991)]. This phenomenon was first considered as the hallmark of flow instability due to a phase transition taking place in the bulk [Berret et al. (1994)]. Observations of the strain field under flow [Cappelaere et al. (1995); Britton and Callaghan (1997); Salmon et al. (2003)] then demonstrated that the second phase appears at the beginning of the stress plateau, in a band close to the inner cylinder; as the apparent shear rate increases along this plateau, this band grows up until filling the gap; at any time there is a discontinuity in shear rate at the interface between the two bands.

These examples show the interest of velocity profile measurements in order to ascertain the origin of the apparent behavior of complex fluids. In this aim various techniques may be used, i.e., light scattering, particle imaging velocimetry, ultrasounds, magnetic resonance imaging, etc., which interpret a physical signal in terms of local velocity. Here we propose an alternative, original method that does not require any sophisticated setup but only a rheometer. The principle is as follows. For a fluid flowing under controlled stress within a wide-gap Couette system, one can reconstruct the velocity profile from a series of similar tests but under smaller stress amplitudes. This is possible because the shape of the stress distribution does not vary with the applied torque level, so that the local rotation velocity at some finite distance within the material for a given torque level is identical to the rotation velocity of the inner cylinder for a sufficiently smaller torque applied. Although the principle of this approach is very simple, its underlying theory needs to be rigourously established in order to determine its field of validity and to use it for more complex torque histories than creep tests such as torque ramps or oscillations. The complete theoretical bases of this method, which only requires a rather general form of the constitutive equation of the material, are presented in Sec. II. In practice, this technique appears to be particularly suitable for yield stress fluids and might be used for detecting wall slip. We carried out experiments in order to validate the technique and illustrate some of its possibilities. In Sec. III, we present the materials and experimental procedures (rheometry and MRI). In Sec. IV, we compare the velocity profile obtained by this technique from that determined by MRI rheometry with a model material. In Sec. V, we show and discuss the results obtained with two pasty materials and with two different types of tool surfaces. 


\section{THEORY}

\section{A. Generalities}

Let us consider the flow of a material between coaxial cylinders of inner and outer radii, respectively, equal to $r_{1}$ and $r_{2}$. We assume that only the inner cylinder can rotate and $r_{2} \rightarrow \infty$. We describe the fluid motion in the cylindrical frame of reference $(O, r, \theta, z)$ fixed to the outer cylinder. For a homogeneous and isotropic material, if inertia and edge effects are negligible, it results from symmetry considerations that in the absence of any flow instability the only nonzero component of the velocity is the tangential one, i.e., $v=r \omega$, which only depends on time and distance from the axis. In the following theoretical approach we will mainly consider the evolution of $\omega=\omega(r, t)$, the rotation velocity within the material at the distance $r$ from the axis and at the time $t$. With a viscous fluid we have $\omega\left(r_{2} \rightarrow \infty\right)=0$, so that in the absence of wall slip the rotation velocity $\Omega(t)$ of the inner cylinder at the time $t$ may be written as

$$
\Omega(t)=-\int_{r_{1}}^{\infty}\left(\frac{\partial \omega}{\partial r}\right) d r .
$$

Using the definition of the local, instantaneous, shear rate, i.e., $\dot{\gamma}(r, t)=-r \partial \omega / \partial r$, Eq. (1) becomes

$$
\Omega(t)=\int_{r_{1}}^{\infty} \frac{\dot{\gamma}}{r} d r
$$

On the basis of the above assumptions, the momentum equation in the tangential direction gives the shear stress distribution at any time within the material:

$$
\tau(r, t)=\frac{C}{2 \pi h r^{2}},
$$

in which $h$ is the length of fluid in contact with the cylinder axis and $C=C(t)$, the torque applied to the inner cylinder at the time $t$.

From the principles of determinism and local action [Coleman et al. (1966)], it may be demonstrated that the behavior of an incompressible, homogeneous, simple material in simple shear can be expressed in the form of a relationship between the shear stress and the history of the local shear:

$$
\tau(r, t)=F_{t^{*}<t}\left[\dot{\gamma}\left(r, t^{*}\right)\right]
$$

in which $F$ is a functional. The normal stress differences may also be expressed with formally similar relationships as a function of the shear rate history. As a consequence, the current shear rate a priori depends on the history of both the shear stress and the normal stress differences. However, intuitively, it seems that the flow characteristics should only depend on the unique boundary condition in terms of stress, i.e., the torque applied. Here, following this idea, we will assume that the normal stress differences only depend on the history of $C(t) / r^{2}$. This might, in fact, constitute a general case in this particular context since any elementary solution of the type $\sigma_{r r}=\alpha C(t) / r^{2}$; $\sigma_{\theta \theta}=-\alpha C(t) / r^{2}$ appears to be a solution of the momentum equation in the radial direction.

Under these conditions the shear rate finally depends solely on the history of $C(t) / r^{2}$, which we write as 


$$
\dot{\gamma}(r, t)=G_{t^{*}<t}\left[\tau\left(r, t^{*}\right)\right],
$$

in which $G$ is a functional. We can rewrite Eq. (5) by using a state of reference, $S_{0}$, reached at the initial time $(t=0)$ :

$$
\dot{\gamma}(r, t)=G_{0}<t^{*}<t\left[S_{0} ; \tau\left(r, t^{*}\right)\right] .
$$

In Eq. (6), $S_{0}$ is defined as the distribution of local, instantaneous, shear rate and the state of structure in the material. In the present context it is not necessary to further define $S_{0}$ and the state of structure.

\section{B. Creep tests}

Here, we assume that from the initial time a constant torque is applied to the inner cylinder, so that

$$
t^{*}>0, \tau\left(r, t^{*}\right)=\frac{C}{2 \pi h r^{2}}=\tau(r)
$$

It follows from Eqs. (6) and (7) that we can make a change of variable $(r \leftrightarrow \tau)$ in Eq. (2), which leads to

$$
\Omega(t)=\frac{1}{2} \int_{0}^{\tau_{1}} \frac{\dot{\gamma}(\xi, t)}{\xi} d \xi
$$

in which $\tau_{1}=C / 2 \pi h r_{1}^{2}$ is the shear stress along the inner cylinder. Our calculations of the rotation velocity starting from Eq. (1) could have been done for any distance $r$ within the material, thus leading similarly to

$$
\omega(r, t)=\frac{1}{2} \int_{0}^{\tau(r) \dot{\gamma}(\xi, t)} \frac{.}{\xi} d \xi .
$$

Although apparently rather simple, Eq. (9) is fundamental since it shows that for a given torque and a given initial state the rotation velocity at any distance and time is given by a similar integral of the same function, whatever the radius of the inner cylinder. This means that for this particular geometry the local rotation velocity only depends on the local torque history. More precisely, Eq. (9) implies that the rotation velocity $\omega(r, t)$ corresponding to an applied torque $C$, is equal to the rotation velocity of the inner cylinder for an applied torque $C^{*}$, such that $\tau(r)_{C}=\tau\left(r_{1}\right)_{C} *$, i.e., $C^{*}=\left(r_{1} / r\right)^{2} C$. This results in the distribution of $\left\{\omega_{C}(r, t) ; r>r_{1}\right\}$ corresponding to the distribution $\left\{\Omega_{r_{1}}\left(C^{*}, t\right) ; C^{*}<C\right\}$, which means that the spatial distribution of rotation velocities may be obtained from the ensemble of rotation velocities of the inner cylinder under different, smaller torques, starting from the same initial state.

\section{Torque variations in time: Torque ramp or controlled stress oscillations}

Since in the stress expression (3) time and distance are separated variables, the change of variable in Eq. (2) can be made for any type of time dependence of the torque, which means that Eq. (9) is always valid. As a consequence, we can proceed to the same reconstruction if we can reproduce conditions for which $\dot{\gamma}(\xi, t)$ remains uniquely defined, i.e., as soon as the stress history at a distance $r$ under the torque $C$ and at a distance $r_{1}$ under a torque $C^{*}$ are similar, which we writes as 


$$
t^{*}>0, \quad C^{*}\left(t^{*}\right)=\left(r_{1} / r\right)^{2} C\left(t^{*}\right)
$$

Let us consider the case of a linear ramp of torque applied to the material, i.e., the torque at the time $t$ is given as

$$
C(t)=2 \pi h K t,
$$

in which $K$ is a constant. We deduce that the distribution of $\left\{\omega_{K}(r, t) ; r>r_{1}\right\}$ is equal to the distribution $\left\{\Omega_{r_{1}}\left(K^{*}, t\right) ; K^{*}<K\right\}$ with $K^{*}=\left(r_{1} / r\right)^{2} K$, i.e., the spatial distribution at a given time during a torque ramp may be found from an ensemble of rotation velocities of the inner cylinder under torque ramps at smaller rates (of stress increase), starting from the same initial state.

A similar approach may be used for dynamics tests under controlled torque. If the torque at the time $t$ is given as:

$$
C=A \sin f t,
$$

in which $f$ is the frequency, we deduce that the distribution of $\left\{\omega_{A}(r, t) ; r>r_{1}\right\}$ is equal to the distribution $\left\{\Omega_{r_{1}}\left(A^{*}, t\right) ; A^{*}<A\right\}$ with $A^{*}=\left(r_{1} / r\right)^{2} A$, i.e., the spatial distribution at a given time during torque oscillations may be found from an ensemble of rotation velocities of the inner cylinder under torque oscillations at smaller amplitudes but under the same frequency, starting from the same initial state.

\section{Finite outer cylinder radius and yield stress fluids}

The correspondence of the velocity distribution within the gap with the set of rotation velocities obtained under various stress magnitudes results, among others, from the fact that the lower boundary of the integrals in Eq. (8) or (9) remains constant: it is equal to zero, which derives from the assumption of infinite outer radius. Thus, in practice, this technique a priori only provides an approximate velocity profile, which is closer to the effective profile as the ratio $r_{1} / r_{2}$ decreases to zero. However, there is a specific case for which our results remain strictly valid though $r_{2}$ is finite. Indeed, if at any time $t$, for the flow under study, we have

$$
\tau<\tau_{2} \Rightarrow \dot{\gamma}(\tau, t)=0
$$

in which $\tau_{2}=C / 2 \pi h r_{2}^{2}$ is the shear stress along the outer cylinder, and we get

$$
\omega(r, t)=\frac{1}{2} \int_{\tau_{2}}^{\tau(r)} \frac{\dot{\gamma}(\xi, t)}{\xi} d \xi=\frac{1}{2} \int_{0}^{\tau(r)} \frac{\dot{\gamma}(\xi, t)}{\xi} d \xi .
$$

Since Eq. (13) would remain valid for any lower stress applied, Eq. (14) remains valid in that case and we can again demonstrate that under creep tests, torque ramps, or oscillations, the velocity distribution within the gap is given by the ensemble of inner cylinder velocities under smaller torque levels.

The situation described by Eq. (13) is generally considered to occur for yield stress fluids when the applied stress along the outer cylinder is, at least in steady state, i.e., for sufficiently large values of $t$, smaller than the yield stress of the fluid. However, the question of the validity of Eq. (13) for such materials under transient flows remains open since their yielding character was shown to be intimately related to their thixotropic character [Coussot et al. (2002b)]. For example, it was shown that, under a fixed stress, the material could flow rapidly during several tenth of seconds before eventually stopping. Subsequent MRI observations of the velocity profiles of bentonite suspensions effectively showed that after a sudden decrease of the rotation velocity of the inner 
cylinder the material can go on flowing for a significant time in a region where the stress is lower than its yield stress in steady state [Raynaud et al. (2002)]. The situation might, nevertheless, be more favorable for start flows: indeed, after a sufficient time of rest, for a sudden increase of the rotation velocity of the inner cylinder, the position of the interface between the sheared and the unsheared regions progressively increases until reaching an asymptotic value $\left(r_{c}\right)$, so that Eq. (13) remains valid if $r_{c}<r_{2}$. Besides these thixotropic effects the possible elastic deformations of the material, which cannot easily be observed by MRI, might concern the whole gap so that Eq. (13) would not be valid at the beginning of the flow.

\section{E. Finite outer cylinder radius and elastic or Newtonian materials}

For a Newtonian fluid, i.e., such that $\tau=\mu \dot{\gamma}$, using Eqs. (2) and (3), the rotation velocity of the inner cylinder can be written:

$$
\omega\left(r_{1}, t\right)=\int_{r_{1}}^{r_{2}} \frac{\dot{\gamma}}{r} d r=\frac{C(t)}{4 \pi h \mu}\left[\frac{1}{r_{1}^{2}}-\frac{1}{r_{2}^{2}}\right]=\left(1-\left(r_{1} / r_{2}\right)^{2}\right) \int_{r_{1}}^{\infty} \frac{\dot{\gamma}}{r} d r .
$$

This means that the rotation velocity of the inner cylinder is simply proportional to that expected in a Couette geometry with an infinite outer radius, a situation for which the above developments are valid. As a consequence, the rotation velocity profiles in the range $\left[r_{1} ; r_{2}\right]$ for a Newtonian fluid [submitted to a torque history of the type $\left.C_{0} f(t)\right]$ in a Couette system with infinite outer radius, can be deduced from a set of experiments under smaller torque amplitudes in a Couette geometry with finite outer radius. The former profile is obtained by dividing the measured rotation velocities by a factor [ 1 $\left.-\left(r_{1} / r_{2}\right)^{2}\right]$. For an elastic material, i.e., such that $\tau \propto \gamma$, a similar result may be obtained concerning the profile of rotation angles. As a consequence, this result is also valid for a viscoelastic solid following a constitutive equation of the form $\tau=G \gamma+\mu \dot{\gamma}$, in which $G$ is a material parameter. This is, in particular, interesting in view of observing the strain field of pasty materials around the solid-liquid transition.

\section{F. Wall slip effect}

The above developments are valid only if the local motion is at any time strictly associated with a flow of the homogeneous material. However, different phenomena such as fracture, wall slip, density heterogeneities, etc., may affect the reconstructed profile in an a priori unknown way. At least some of them should be detectable from a thorough analysis of the results. Let us, for example, consider the case of wall slip. We assume that it only affects a layer of negligible thickness close to the inner cylinder and induces an additional rotation velocity of the inner cylinder, $\Omega_{S}$, which a priori depends on the local shear stress $\left(\tau_{1}\right)$ and on the time. Under these conditions Eq. (9) becomes

$$
\Omega(t)=\Omega_{S}\left(\tau_{1}, t\right)+\frac{1}{2} \int_{0}^{\tau_{1}} \frac{\dot{\gamma}(\xi, t)}{\xi} d \xi
$$

while Eq. (3) remains unchanged. This results in the distribution of $\left\{\omega_{C}(r, t) ; r>r_{1}\right\}$ within the material now being equal to the distribution $\left\{\Omega_{r_{1}}\left(C^{*}, t\right)-\Omega_{S}\left(C^{*}, t\right) ; C^{*}\right.$

$<C$ \}. Thus, the reconstruction technique may be used in the presence of wall slip as long as one is capable of quantifying the effect of wall slip from independent measurements. 
Conversely, if we a priori ignore wall slip although it effectively occurs, and directly compute the distribution $\left\{\Omega_{r_{1}}\left(C^{*}, t\right) ; C^{*}<C\right\}$, we will, in fact, get the velocity distri-

bution $\left\{\omega_{C}(r, t)+\Omega_{S}\left[\left(r_{1} / r\right)^{2} C, t\right] ; r>r_{1}\right\}$. For pasty materials, the slip effect is dominant only at low stresses and seems to follow a power-law model at stresses smaller than the fluid yield stress [Aral and Kalyon (1994); Bertola et al. (2003)]. It follows that in practice wall slip could be detected, at least qualitatively, from an apparent velocity profile composed of two regions: close to the wall a region of rapidly decreasing velocity profile, in which the shear-thinning character of the bulk fluid dominates $\left(\omega_{C} \gg \boldsymbol{\Omega}_{S}\right)$, and a region of much slower decrease in velocity farther from the inner cylinder, in which the slip velocity term dominates $\left(\omega_{C} \ll \boldsymbol{\Omega}_{S}\right)$.

\section{EXPERIMENTAL PROCEDURES}

\section{A. Materials}

We used different types of material:

- A polymeric suspension: a commercial hair gel (May gel "normal," made by Yplon S. A., Belgium), basically made of Carbopol and extremely stable under various conditions; and

- Two pasty suspensions: a kaolin-water mixture (Kaolins de Beauvoir, Echassières, France) with a solid volume fraction of $23.5 \%$, and a mustard (Amora, France)

\section{B. Rheometry}

We used a stress controlled Paar Physica MC1+ system equipped with a large gap, coaxial cylinder geometry (inner cylinder diameter: $2.5 \mathrm{~cm}$; outer cylinder diameter: 4.9 $\mathrm{cm})$. In order to avoid wall slip, the tool surfaces were roughened: the outer cylinder was covered with sandpaper (roughness: $0.5 \mathrm{~mm}$ ), and the inner cylinder was striated (roughness: $2 \mathrm{~mm}$ ). One experiment was also carried out with smooth surfaces (steel). After their preparation the materials were introduced in the rheometer and presheared at 300 rpm during a fixed time then left at rest during another time, and a given level of torque was applied during a time ranging from 2 and $10 \mathrm{~min}$. Successive torque levels were then applied in the same way following the same procedure (preshear, rest, shear), which ensures that the initial state of the material $\left(S_{0}\right)$ was identical before each creep test. The different torque levels were chosen in order to obtain a sufficient resolution of the reconstructed velocity profile. The tests were carried out at a temperature of $20^{\circ} \mathrm{C}$. In order to avoid drying effects, the geometry was covered with a paraffine film. It was checked that no significant evolution of edge effects or material characteristics occurred by comparing the torque levels reached during the different preshears. When the sample was removed during a series of creep tests in order to avoid its possible degradation in time, we used the torque level under the first preshear as a reference to correct the new effective torque, which makes it possible to take into account slight changes of sample volume or edge effects.

\section{Magnetic resonance imaging}

As a "MRI rheometer" we used a setup and procedures described in detail by Raynaud et al. (2002). Here, we only recall the main aspects. We used a vertical coaxial cylinder geometry (inner cylinder radius: $r_{i}=4 \mathrm{~cm}$; outer cylinder of radius $r_{e}$ $=6 \mathrm{~cm}$; length: $h=11 \mathrm{~cm}$ ). The surfaces of the outer and inner cylinder in contact with the fluid were covered with a sandpaper with an equivalent roughness of $200 \mu \mathrm{m}$. Only the rotation velocity of the inner cylinder can be controlled. The temperature of the 
material in the Couette geometry could not be controlled but the room temperature was kept between 20 and $24^{\circ} \mathrm{C}$. Magnetic resonance imaging was performed with a Bruker (France) setup equipped with a vertical $0.5 \mathrm{~T}$ magnet (Magnex Scientific, Ltd., U.K.) fitted with shielded gradients leaving a free bore of $25.5 \mathrm{~cm}$ and delivering a gradient of $50 \mathrm{mT} / \mathrm{m}$ with a rise time of $500 \mu \mathrm{s}$. The signal was collected within a linear birdcage coil of $24 \mathrm{~cm}$ length and $20 \mathrm{~cm}$ diam. We used a sequence derived from that of Hanlon et al. (1998): a one-dimensional acquisition was carried out with a single spin-echo sequence where $180^{\circ} \mathrm{rf}$ selectivity was moved from the slice selection axis to another axis in order to select a rectangular volume of interest and enhance signal to noise by accumulation. The volume imaged was a (virtual) rectangular portion of $30 \mathrm{~mm}$ in the axial direction with a width (in the tangential direction) of $10 \mathrm{~mm}$ and a length of $70 \mathrm{~mm}$ (in the radial direction, starting from the central axis), but only velocity data from the rheometer gap (filled with the sample) are relevant and presented here. This volume was situated at the magnet center (so as to damp the effects of field heterogeneities), and sufficiently far from the bottom and the free surface of the rheometer for flow perturbations due to edge effects remaining negligible (this could be checked by velocity imaging at different depths in the fluid). This approach has been validated from tests with materials rotating as a plug within a single, rotating cylinder, and from tests with a Newtonian fluid [Raynaud et al. (2002)]. The spatial resolution of our measurements is $0.5 \mathrm{~mm}$. Only tests with the polymeric gel were carried out. With this material no density heterogeneity can develop under usual flow conditions. The uncertainty of the velocity data was estimated theoretically from the amplitude of electromagnetic and electronic noise. For the test presented here, it was approximately equal to $5 \times 10^{-6} \mathrm{~m} \mathrm{~s}^{-1}$ for all points in the gap.

\section{RESULTS AND DISCUSSION}

\section{A. Validation of the reconstruction method}

In order to validate our reconstruction procedure, we carried out MRI-rheometrical tests and velocity reconstruction tests with the laboratory rheometer on the same material (polymeric gel). The velocity profile for a given torque level, reconstructed from a series of creep tests after 10 min of flow with this gel, is shown in Fig. 1. The rotation velocity used in correspondence with the different stress values was obtained by averaging the velocity over the last minute of flow. The experimental procedure with the MRI rheometer was roughly similar to that used, at a smaller scale, with the laboratory rheometer, i.e., after its insertion within the geometry the material was presheared during $10 \mathrm{~min}$ at $130 \mathrm{rpm}$, then left or not at rest during $5 \mathrm{~min}$, and sheared at a given velocity. The velocity profiles obtained with or without preliminary rest were almost perfectly similar, which indicates that over the duration of averaging $(10 \mathrm{~min})$ transient effects are negligible. Since, with the MRI rheometer, this is the rotation velocity that is imposed, in view of our comparison between the two techniques, we fixed it to that obtained with the laboratory rheometer under the chosen torque. Note that with MRI a single test of limited duration $(10 \mathrm{~min})$ was sufficient to obtain the complete velocity profile at this rotation velocity.

The corresponding MRI velocity profile is shown in Fig. 1. By repeating this test we could check that the data were perfectly reproducible within the mean theoretical uncertainty on our measurements, i.e., $\Delta v / r_{1} \approx 1.2 \times 10^{-4} \mathrm{~s}^{-1}$. Note that beyond $r / r_{1}$ $=1.4$ the values of $v / r_{1}$ appear to fluctuate in a slightly larger range: $\lfloor-2$ $\times 10^{-4} ; 10^{-4} \mathrm{~s}^{-1} \mathrm{~J}$. It is worth noting that in linear scale (see the inset in Fig. 1) there clearly appears a strong discontinuity of the slope of the velocity profile at the approach 


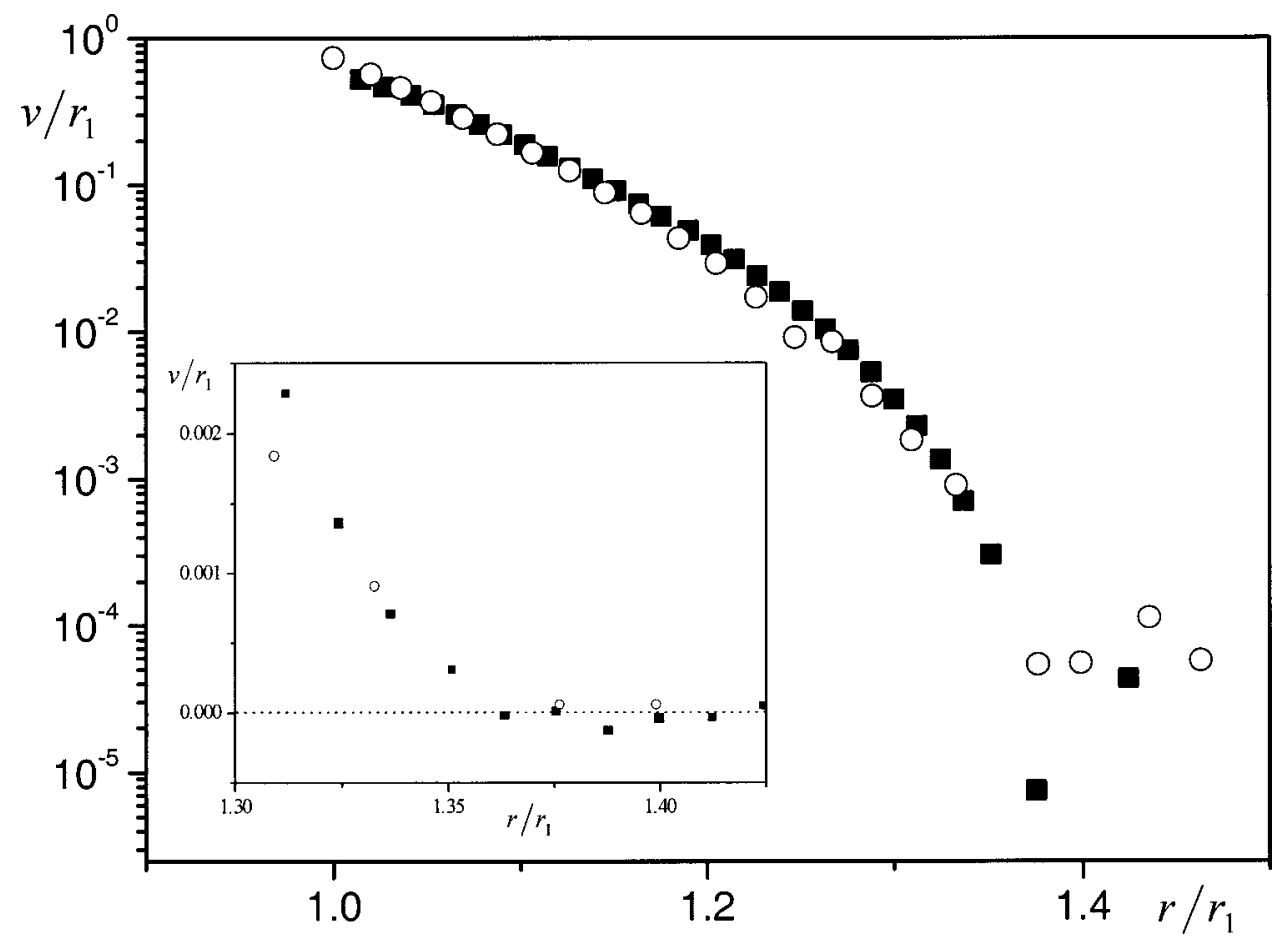

FIG. 1. Steady-state velocity profile for the polymeric gel in the Couette geometry for a given rotation velocity, as reconstructed from creep tests (circles) and measured by MRI (squares). The velocity to inner cylinder radius ratio is expressed in $\mathrm{s}^{-1}$. The inset shows at a larger scale the data around the interface between the sheared and the (apparently) unsheared region.

of the abscissa axis, which indicates that the rheological behavior of this fluid is qualitatively similar to that of various pasty materials exhibiting a viscosity bifurcation around a critical stress (see Sec. I).

Since both techniques show that there is no apparent flow beyond a critical distance within the gap, our reconstruction procedure can be applied relevantly (see Sec. IIE). Note that for the comparison between the data obtained from the two different rheometers, we scaled the tangential velocity and the distance by the radius of the inner cylinder. Indeed, a simple dimensional analysis [Raynaud et al. (2002)] shows that in such a representation the velocity profiles obtained for the same material in two Couette geometries of different inner diameters are, in theory, similar as long as there is an unsheared region within the gap in both cases. Although there remains some slight discrepancy, which is likely due to the uncertainty on both techniques at small velocities, the two velocity profiles are almost exactly similar in a logarithmic scale over four decades of velocities, which proves the validity of both techniques. More generally, this shows that as long as the material remains homogeneous, a thorough analysis and interpretation of rheometrical data obtained from experiments under controlled stress can be put in perfect correspondence with direct velocity measurements.

\section{B. Interpretation of transient flows under creep tests in terms of velocity profiles}

We carried out a series of creep tests with the mustard and the kaolin suspension. In both cases the diagram of rotation velocities as a function of time for the different stress 
Torque (mN.m):

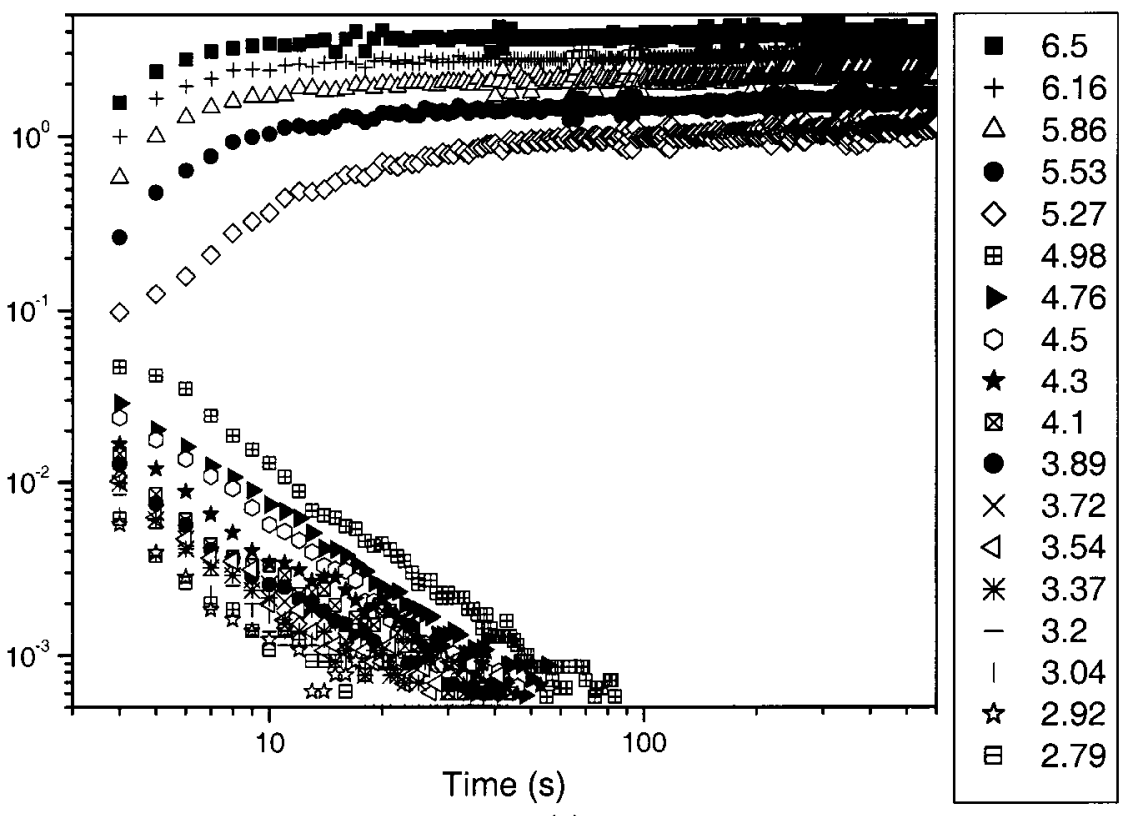

(a)

Torque (mN.m):

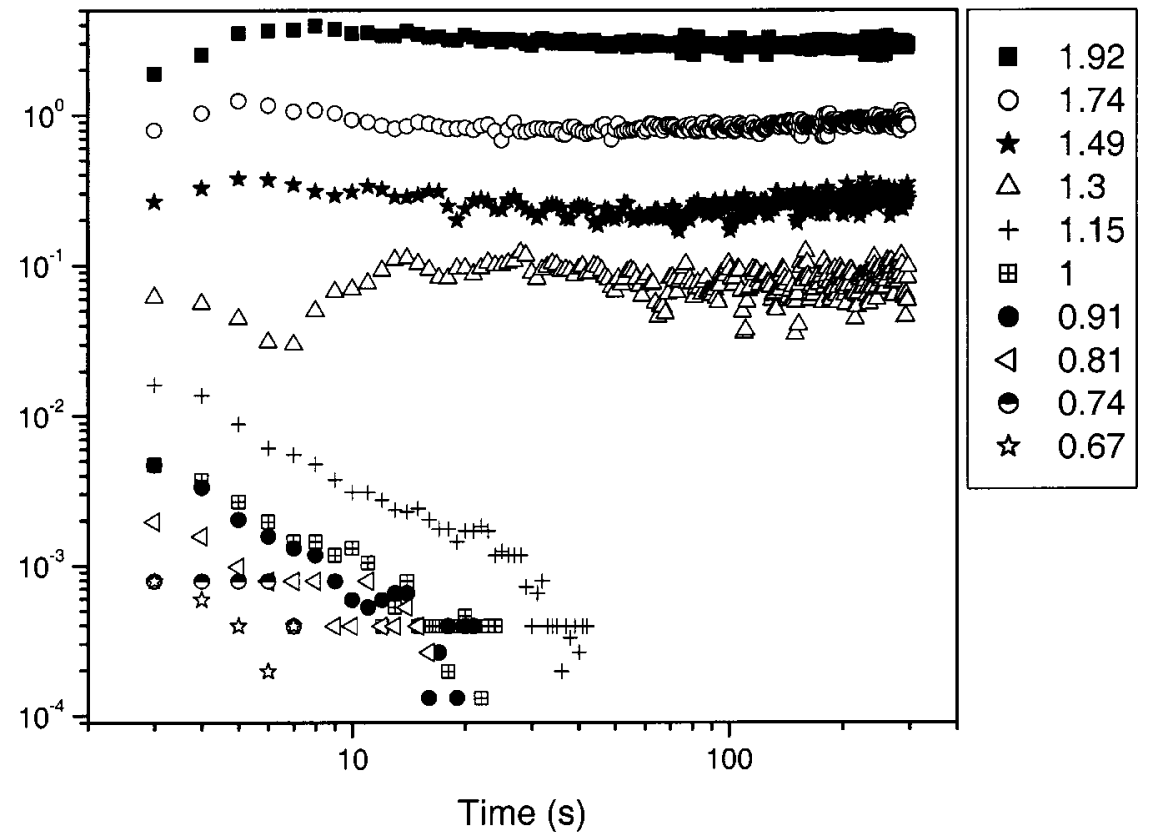

(b)

FIG. 2. Creep tests: rotation velocity as a function of time for different torque levels following preshear at 300 rpm and rest. Mustard (a) 5 min preshear, 1 min rest; Kaolin suspension (b) 2 min preshear, 1 min rest. 
levels (cf. Fig. 2) exhibits the "viscosity bifurcation" phenomenon already observed for various other materials [Coussot et al. (2002a)]: beyond a critical stress level the velocity initially increases then tends to a steady-state value; below this critical level the velocity progressively decreases towards zero. Under such conditions the reconstruction of the velocity profiles in time (cf. Fig. 3), using the procedure of Sec. II B, is particularly interesting. During the first instants the fluid is apparently sheared over a long distance, then there is a progressive separation of the fluid into two regions: close to the inner cylinder the fluid goes on flowing at a significant rate while the velocity profile in the rest of the fluid progressively tends to a plateau, the level of which decreases towards zero. It is worth noting that the complete reliability of the velocity profiles determined in the first instants is doubtful since the material appears to be sheared over all the gap. Nevertheless, we can at least qualitatively interpret this as an elastic regime. Eventually, after a certain time, in a logarithmic scale, the velocity profile appears to be broken at a critical distance: the velocity in the region far from the inner cylinder is several decades smaller than in the other region. In this regime our reconstruction technique is fully relevant. In a linear scale the velocity profile in steady state appears to have a clear discontinuity in its slope at the approach of the abscissa axis (see, for example, Fig. 4). This aspect is similar to that found for other pasty materials from MRI rheometry [Coussot et al. (2002)]. To sum up, this technique makes it possible to establish a straightforward correspondence between the viscosity bifurcation effect observed with usual rheometers and the velocity profiles measured by nuclear magnetic resonance and exhibiting a slope discontinuity.

\section{Effect of wall slip}

We carried out similar tests with the mustard with a smooth inner cylinder and reconstructed the velocity profiles in steady state (Fig. 4). In both cases the velocity profiles were determined by averaging the rotation velocity over the last $30 \mathrm{~s}$ of a 2 min test. Apart from a systematic difference in the position of the velocity profile, which may be explained in part by a shift due to the slip velocity, the results are basically similar in the sheared region but strongly differ in the unsheared region. With the smooth surfaces the velocity stabilizes at an almost perfectly, constant level in the region associated with the absence of motion with rough surfaces. This result is qualitatively in agreement with the theoretical predictions (Sec. II F): the reconstructed velocity distribution in the sheared region is not significantly affected by wall slip but the slip velocity is superimposed and becomes apparent in the unsheared region. The constancy of the corresponding velocity level implies that $r \Omega_{S}\left[\left(r_{1} / r\right)^{2} C\right]$ remains constant in our range of distances, which suggests that for this material the rotation velocity due to slip approximately increases as the root of the stress.

\section{CONCLUSION}

We proposed a new technique of reconstruction of the velocity profiles during tests under controlled stress. This technique has various advantages: it only requires a rheometer and its limits in terms of spatial and time resolutions only depend on those of the rheometer and the patience of the experimenter. Its disadvantages are that obtaining a complete series of velocity profiles in time with good resolution for a single test requires several hours. Moreover, this approach is valid only if there is an unsheared region far from the inner cylinder, a hypothesis which, in practice with a finite gap, can only be checked a posteriori. Finally, since this is an indirect method of observation it may be affected by some perturbating effects such as wall slip or fracture, which can nevertheless be detected by an analysis of the shape of the velocity profiles. 


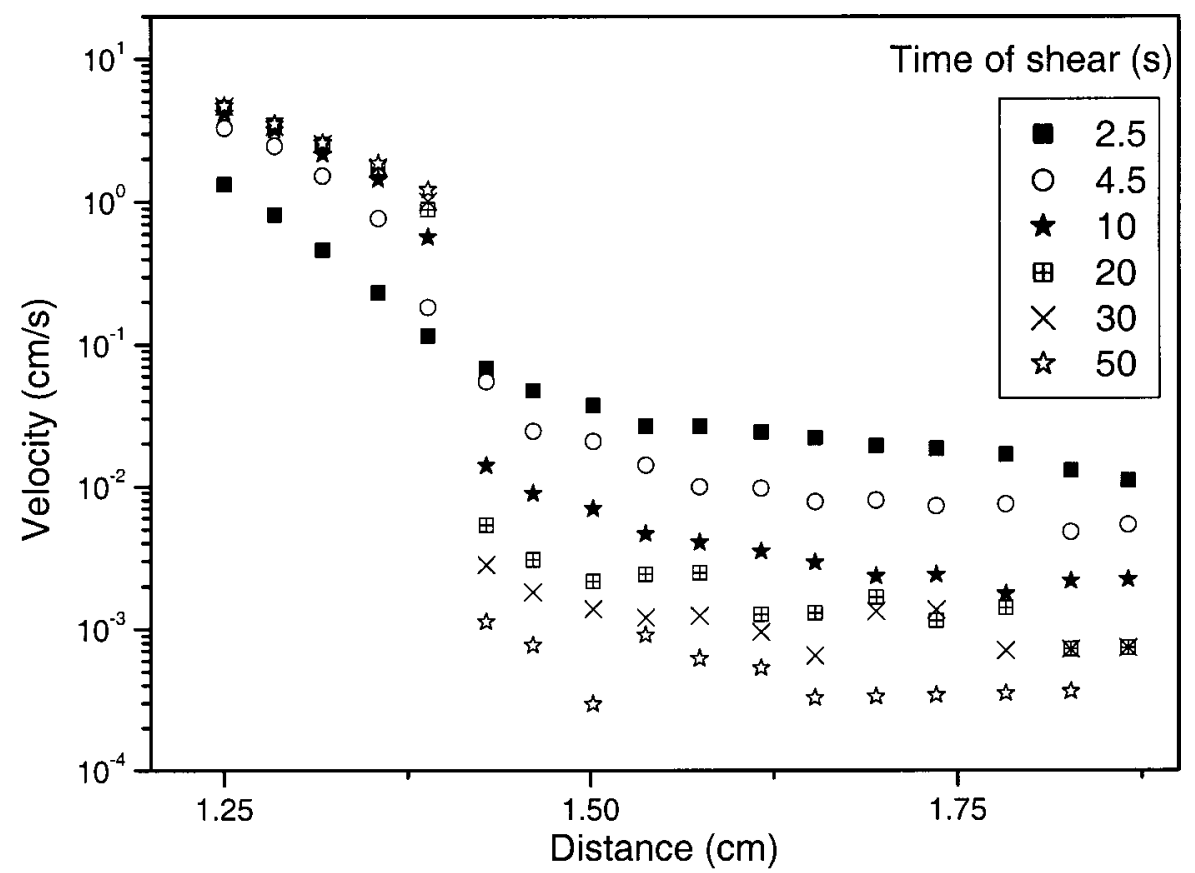

(a)

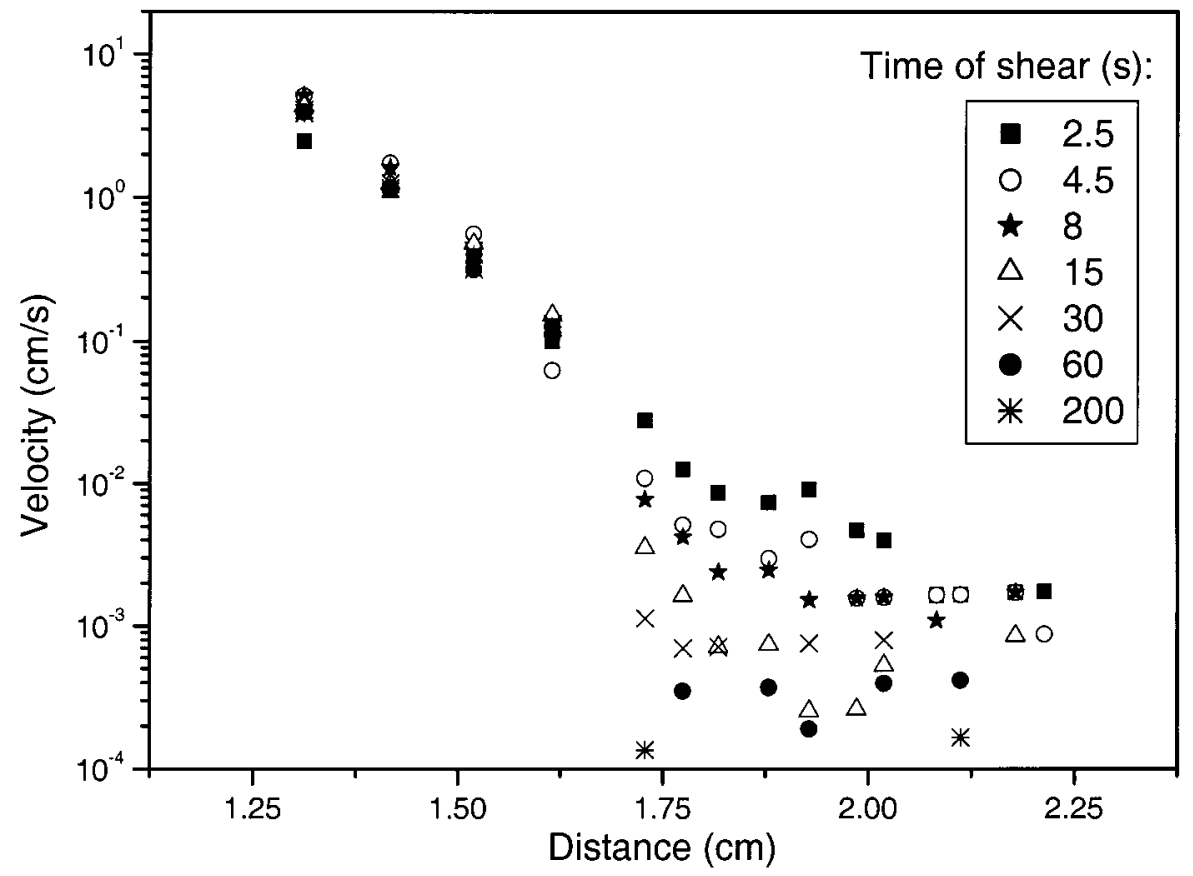

(b)

FIG. 3. Velocity profiles in time under a given torque level as reconstructed from the creep tests of Fig. 2: Mustard (a) $6.5 \mathrm{mN} \mathrm{m}$; Kaolin suspension (b) $1.92 \mathrm{mN} \mathrm{m}$. 


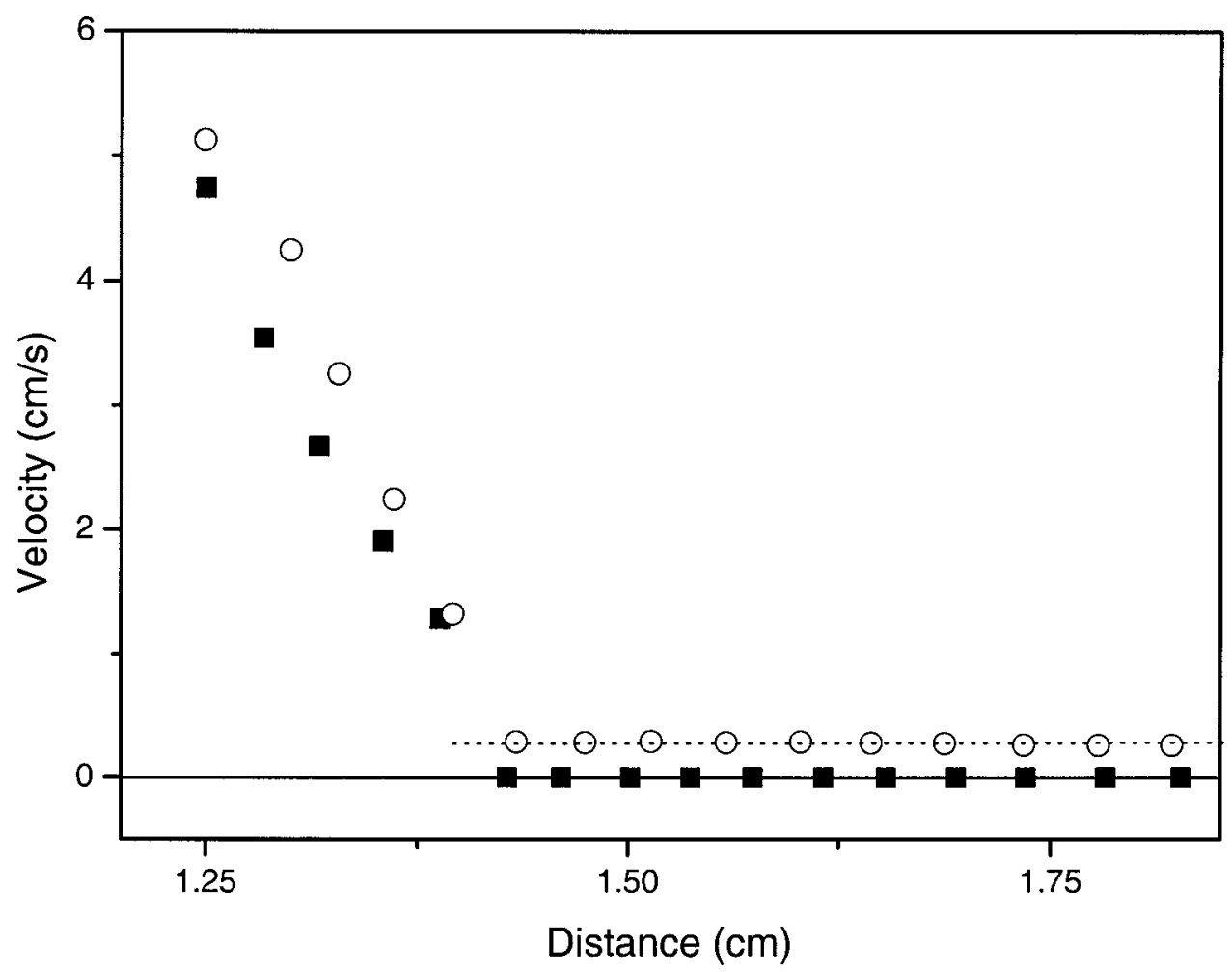

FIG. 4. Reconstructed velocity profile for the mustard in steady state under a torque of $1.92 \mathrm{mN} \mathrm{m}$ with rough (squares) and smooth (circles) surfaces.

It remains that this technique is of strong interest in that it makes it possible to interpret, in a straightforward way, in terms of velocity distribution in the gap, the data from simple tests under controlled stress. We can thus have access to local characteristics of the flow from usual macroscopic measurements, which seems extremely useful for viscoelastic, yielding, or thixotropic fluids, for which the regularity of the flow in the gap is sometimes questionable. For example, this technique already made it possible to establish a clear link between the viscosity bifurcation effect observed from usual creep tests and the slope rupture in velocity profiles observed by MRI for pasty materials. Moreover, although it has been validated here from a comparison of steady-state flow data (due to MRI limitations), it may be used to study the transient flow properties of complex fluids in relation to the evolution of their internal structure over extremely short times.

\section{References}

Aral, B. K., and D. M. Kalyon, "Effects of temperature and surface roughness on time-dependent development of wall slip in steady torsional flow of concentrated suspensions," J. Rheol. 38, 957-972 (1994).

Berret, J. F., D. C. Roux, G. Porte, and P. Lindner, "Shear-induced isotropic-to-nematic phase transition in equilibrium polymers," Europhys. Lett. 25, 521-527 (1994).

Bertola, V., F. Bertrand, H. Tabuteau, D. Bonn, and P. Coussot, "Wall slip and yielding in pasty materials," J. Rheol. 47, 1211-1226 (2003). 
Britton, M. M., and P. T. Callaghan, “Two-phase shear band structures at uniform stress," Phys. Rev. Lett. 78, 4930-4933 (1997).

Cappelaere, E., R. Cressely, and J. P. Decruppe, "Linear and nonlinear rheological behavior of salt-free aqueous CTAB solutions," Colloids Surf., A 104, 353-374 (1995).

Coleman, B. D., H. Markowitz, and W. Noll, Viscometric Flows of Non-Newtonian Fluids (Springer, Berlin, 1966).

Coussot, P., J. S. Raynaud, F. Bertrand, P. Moucheront, J. P. Guilbaud, H. T. Huynh, S. Jarny, and D. Lesueur, "Coexistence of liquid and solid phases in flowing soft-glassy materials," Phys. Rev. Lett. 88, 218301 (2002).

Coussot, P., Q. D. Nguyen, H. T. Huynh, and D. Bonn, "Avalanche behavior in yield stress fluids," Phys. Rev. Lett. 88, 175501 (2002a).

Coussot, P., Q. D. Nguyen, H. T. Huynh, and D. Bonn, "Viscosity bifurcation in thixotropic, yielding fluids," J. Rheol. 46, 573-589 (2002b).

Da Cruz, F., F. Chevoir, D. Bonn, and P. Coussot, "Viscosity bifurcation in foams, emulsions and granular systems," Phys. Rev. E 66, 051305 (2002).

Hanlon, A. D., S. J. Gibbs, L. D. Hall, D. E. Haycock, W. J. Frith, and S. Ablett, "Rapid MRI and velocimetry of cylindrical Couette flow," Magn. Reson. Imaging 16, 953-961 (1998).

Pignon, F., A. Magnin, and J. M. Piau, "Thixotropic colloidal suspensions and flow curves with minimum: Identification of flow regimes and rheometric consequences," J. Rheol. 40, 573-587 (1996).

Raynaud, J. S., P. Moucheront, J. C. Baudez, F. Bertrand, J. P. Guilbaud, and P. Coussot, "Direct determination by NMR of the thixotropic and yielding behavior of suspensions," J. Rheol. 46, 709-732 (2002).

Rehage, H., and H. Hoffmann, "Viscoelastic surfactant solutions," Mol. Phys. 74, 933 (1991).

Salmon, J. B., A. Colin, S. Manneville, and F. Molino, "Velocity profiles in shear-banding wormlike micelles," Phys. Rev. Lett. (to be published).

Vermant, J., "Large-scale structures in sheared colloidal dispersions," Curr. Opin. Colloid Interface Sci. 6, 489-495 (2001).

Walls, H. J., S. B. Caines, A. M. Sanchez, and S. A. Khan, "Yield stress and wall slip phenomena in colloidal silica gels," J. Rheol. 47, 847-868 (2003). 